\title{
Ligation-assisted endoscopic full-thickness resection with over-the-scope clip targeted for neuroendocrine tumors
}

\author{
Hideki Kobara, Noriko Nishiyama, Shintaro Fujihara, Tsutomu Masaki
}

Kagawa University, Kagawa, Japan

Because most neuroendocrine tumors (NETs) invade the deep submucosal layer [1], small NETs are a kind of tumors indicated for endoscopic full-thickness resection (EFTR) using an over-the-scope clip (OTSC; Ovesco Endoscopy, Tübingen, Germany) [2]. EFTR is a potentially curative procedure compared with conventional methods that resect only the submucosal layer. However, a new FTR device (Ovesco Endoscopy) [3] is unavailable worldwide. We herein describe a novel ligationassisted EFTR using the original OTSC (1-FTRO) to target small NETs in an animal experiment using a beagle dog (Video 1).

First, a circumferential incision was made around the marking dots in the lower rectum until the muscle layer appeared (First step, Incision: Fig. 1A). Next, after firing the O-ring (endoscopic variceal ligation device, EVL; MD-48720U, Sumius, Tokyo, Japan) directly beneath the prepared incision line (Second step, EVL: Fig. 1B), we constricted a thin snare (Snare Master, SD221L-25; Olympus) below the ring (Third step, Snaring: Fig. 1C). While aspirating the snare into the cap, the OTSC was deployed (Fourth step, OTSC: Fig. 1D). Finally, EFTR with the snare was achieved using an electric generator (VIO300D; Endo Cut Q mode, ERBE, Germany) (Fifth step, EFTR: Fig. 1E). The total procedure time was $16 \mathrm{~min}$, and histological examination of an en bloc specimen (Fig. 2A) confirmed an adequate full-thickness layer (Fig. 2B). The advantages are follows: The incision enables the muscle layer to be identified, the O-ring prevents the snare from slipping, and preparing the snare before OTSC deployment ensures complete EFTR. Therefore, l-FTRO may be reliable for treating small NETs.

Video 1 EFTR is a therapeutic option indicated for small NETs infiltrating the submucosa. This video shows that ligationassisted EFTR with the original OTSC (1-FTRO) enables reliable full-thickness resection without the snare slipping EFTR, endoscopic full-thickness resection; NET, neuroendocrine tumor; OTSC, over-the-scope clip

Departments of Gastroenterology and Neurology, Faculty of Medicine, Kagawa University, Kagawa, Japan (Hideki Kobara, Noriko Nishiyama, Shintaro Fujihara, Tsutomu Masaki)

Conflict of Interest: None

Correspondence to: Hideki Kobara, MD, PhD, Department of Gastroenterology and Neurology, Faculty of Medicine, Kagawa University, 1750-1 Ikenobe, Miki, Kita, Kagawa 761-0793, Japan, e-mail: kobara@med.kagawa-u.ac.jp

Received 15 January 2020; accepted 21 January 2020 published online 14 March 2020

DOI: https://doi.org/10.20524/aog.2020.0468

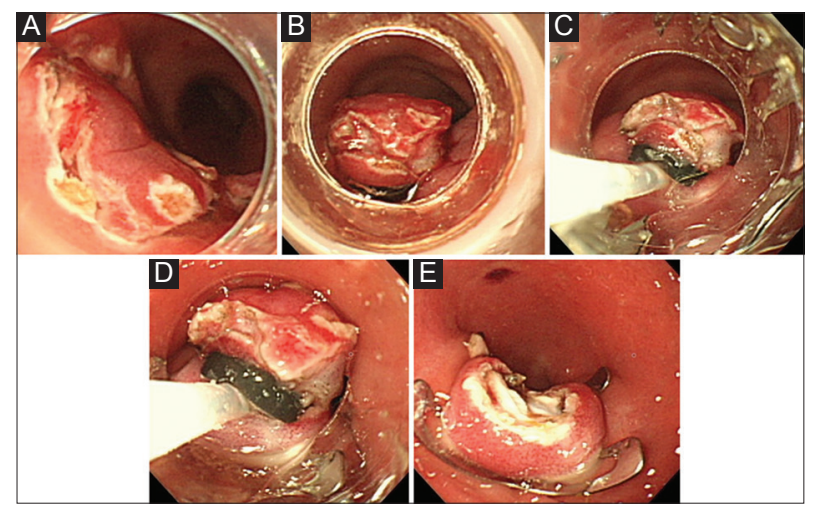

Figure 1 (A) Endoscopic view showing the circumferential incision around the marking dots in the lower rectum (First step, Incision). (B) Endoscopic view showing the O-ring fired directly beneath the prepared incision line (Second step, EVL). (C) Endoscopic view showing the thin snare constricted below the ring (Third step, Snaring). (D) Endoscopic view showing OTSC deployment while aspirating the snare into the cap (Fourth step, OTSC). (E) Endoscopic view showing the completed EFTR with the snare (Fifth step, EFTR)

EVL, endoscopic variceal ligation; OTSC, over-the-scope clip; EFTR, endoscopic full-thickness resection
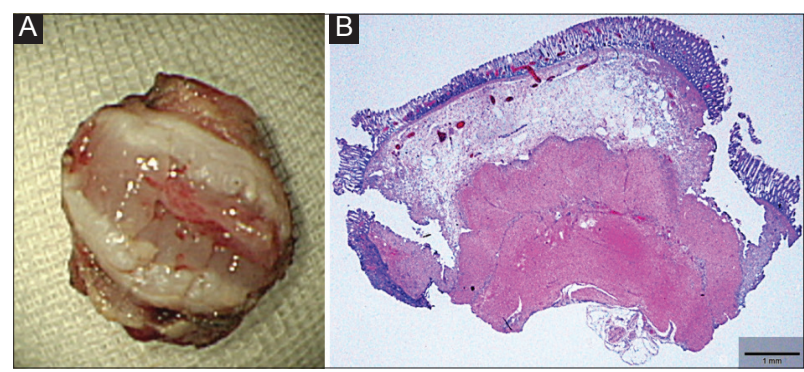

Figure 2 (A) Macroscopic image showing the full-thickness resected specimen. (B) Histological finding revealing the full-thickness layer

\section{References}

1. Zhou X, Xie H, Xie L, Li J, Cao W, Fu W. Endoscopic resection therapies for rectal euroendocrine tumors: a systematic review and meta-analysis. J Gastroenterol Hepatol 2014;29:259-268.

2. Kobara H, Mori H, Nishiyama N, et al. Over-the-scope clip system: A review of 1517 cases over 9 years. J Gastroenterol Hepatol 2018;34:22-30.

3. Schmidt A, Beyna T, Schumacher B, et al. Colonoscopic full-thickness resection using an over-the-scope device: a prospective multicentre study in various indications. Gut 2018;67:1280-1289. 